\title{
A Novel Prediction Method of Dynamic Wall Pressure for Silos Based on Support Vector Machine
}

\author{
Hanhua Yu, ${ }^{1,2}$ Zhijun Xu $\mathbb{D}^{1,2}$ Tingting Liu, ${ }^{1,2}$ and Fang Yuan ${ }^{1,2}$ \\ ${ }^{1}$ College of Civil Engineering, Henan University of Technology, Zhengzhou 450001, China \\ ${ }^{2}$ China National Engineering Laboratory for Grain Storage and Transportation, Zhengzhou 450001, China
}

Correspondence should be addressed to Zhijun Xu; zj.xu_hust@qq.com

Received 5 February 2020; Revised 16 July 2020; Accepted 31 August 2020; Published 8 September 2020

Academic Editor: Binh Thai Pham

Copyright (c) 2020 Hanhua Yu et al. This is an open access article distributed under the Creative Commons Attribution License, which permits unrestricted use, distribution, and reproduction in any medium, provided the original work is properly cited.

The physical properties and mechanical characteristics of storage materials are significantly different from those of ordinary solids and liquids. The distribution of dynamic wall pressure during silo discharge is quite complicated. Considering the nonlinear relationship between the factors which affect the dynamic lateral pressure of silos, a prediction method of dynamic wall pressure for silos based on support vector machine (SVM) is proposed here, and furthermore, the modified grid search method (GSM) is incorporated in obtaining the optimal support vector machine parameters to improve the accuracy of the prediction. Comparing the results of the proposed prediction model with the results of experiment methods and simulation methods, it can be found that the SVM prediction model shows high accuracy and high generalization ability, and the prediction results of the model fit well with the results of experiment and simulation methods. The proposed method can provide reference for the prediction of the dynamic wall pressure of silos.

\section{Introduction}

Silos are widely used to store coals, grains, and gravels because of their small footprint, large capacity, and low cost. The dynamic wall pressure during silo discharge is the main factor that causes silo damage [1]. The investigations about static wall pressure of silos have been relatively perfect $[2,3]$. However, there is no widely accepted theory and calculation method of dynamic wall pressure for silos. Smith and Lohnes proposed the theory of lateral grain expansion [4]. Su proposed the theory of instantaneous arching for grains [5]. Janssen proposed the Janssen theory based on the continuous medium model [6]. These investigations present the theoretical basis for the dynamic wall pressure of silos. Liu and Hao [7] proposed a method for the calculation of dynamic lateral pressure of silos based on the overall flow of stored materials. Yuan et al. [8] proposed a method for the calculation of lateral pressure on squat silo wall by considering the influence of silo diameter ratio, and the methods are mainly used to solve the calculation of lateral pressure of large shallow silos. Based on the unified strength theory and by considering the common influence of three principal stresses, Sun et al. [9] proposed a lateral pressure coefficient which is applied to both the deep and shallow silos. The dynamic lateral pressure of silos is affected by various factors, and the relationships between the influencing factors are nonlinear and complex. Traditional research methods (such as Jassen formula) only consider one or a few factors [10], and it is not conducive to the analysis and research of dynamic lateral pressure. In this way, it is necessary to establish an efficient and simple dynamic lateral pressure prediction method which takes the various factors into consideration. Recently, with the development of artificial intelligence, machine learning has been widely applied in many research fields. As one of the methods of machine learning, support vector machine (SVM) has good generalization performance and is one of the present investigation focus of artificial intelligent algorithms [11]. The SVM is widely used for classification and regression analysis, it is quite efficient in solving the problems about which the characteristics are nonlinear, 
uncertain, small sample, and also, it has been widely applied in civil engineering and shows its efficiency and reliability in such problems. For example, Dai et al. [12] proposed a support vector density-based importance sampling for reliability assessment and showed its efficiency. Also, the least squares support vector machine (LSSVM) technology is applied to the reliability analysis of soft clay foundation settlement in [13]. Wang et al. [14] proposed a support vector machine basing model to predicate the stability coefficient of slope, and the grid search method is used to optimize the parameters.

Thus, considering the efficiency of the support vector machine in solving the problems of complex, nonlinear, multifactor, small sample problems and its advantage in the aspect of prediction, here, the support vector machine method is employed to help the prediction of the dynamic wall pressure for silos. First, the relevant factors affecting the dynamic lateral pressure of silos are set as the input variables, and the dynamic lateral pressure is set as the output value; second, the penalty parameters and kernel function parameters are optimized, respectively, under the help of grid search method, and the prediction model of dynamic lateral pressure of silos is established. Compared with other methods, the proposed model shows its prediction ability in the measurement of dynamic lateral pressure of silos. It provides a new method and basis for calculation and silo design.

\section{Support Vector Machine Principle and Parameter Optimization}

Support vector machine algorithm is based on statistical theory. The advantage of this method is that it adopts the principle of minimizing structural risk. Its model is $[10,15]$

$$
f(x)=\omega \cdot \varphi(x)+b f(x),
$$

where $x$ is the input feature vector; $f(x)$ is the output value; $\varphi(x)$ is the nonlinear function mapped to high-dimensional feature space; $\omega$ is the weighted vector of the hyperplane; and $b$ is the bias vector.

The solution of nonlinear regression is the key point to support vector machine, and it can be solved by using the optimal solution of the following equation:

$$
\min _{w, b} \frac{1}{2}\|w\|^{2}+c \sum_{i=1}^{l}\left(\xi_{i}+\xi_{*}\right)
$$

Equation (2) satisfies the following constraints:

$$
\text { s.t. }= \begin{cases}\left(\omega \cdot x_{i}\right)+b-y_{i} \leq \varepsilon+\xi_{i}, & i=1, \ldots, l, \\ y_{i}-\left(\omega \cdot x_{i}\right)-b \leq \varepsilon+\xi_{i}^{*}, & i=1, \ldots, l, \\ \xi_{i}, \xi_{i}^{*} \geq 0, & i=1, \ldots, l .\end{cases}
$$

where $c$ is the penalty parameter; $\varepsilon$ is the insensitive loss parameter; and $\xi_{i}$ and $\xi_{i}^{*}$ are the slack variables.

The Lagrange formula and Karush-Kuhn-Tucker condition are employed to solve the optimal solution shown in the following equation:

$$
\min _{a, a^{*}} \frac{1}{2} \sum_{i, j=1}^{l}\left(\alpha_{i}^{*}+\alpha_{i}\right)\left(\alpha_{j}^{*}-\alpha_{j}\right)\left(x_{i} \cdot x_{i}\right)+\varepsilon \sum_{i=1}^{l}\left(\alpha_{i}^{*}-\alpha_{i}\right) .
$$

Equation (4) satisfies the following constraints:

$$
\text { s.t. }\left\{\begin{array}{l}
\sum_{i=1}^{l}\left(\alpha_{i}^{*}-\alpha_{i}\right)=0, \\
0 \leq \alpha_{i}, \alpha_{i}^{*} \leq c, i=1, \ldots, l .
\end{array}\right.
$$

where $\alpha_{i}$ and $\alpha_{i}^{*}$ are the Lagrange multipliers.

Kernel function described as $K\left(x_{i}, y_{i}\right)$ is incorporated to solve high-dimensional equations according to support vector machine algorithm. The radial basis function is used as the kernel function due to its satisfactory prediction accuracy, which is shown in the following equation:

$$
K\left(x_{i}, y_{j}\right)=\exp \left(-\frac{\left\|x_{i-} y_{j}\right\|^{2}}{2 \sigma^{2}}\right) .
$$

According to equations (1) and (4)-(6), the regression fitting function of support vector machine can be obtained:

$$
f(x)=\sum_{i=1}^{n s v}\left(\alpha_{i}-\alpha_{i}^{*}\right) K\left(x_{i}, y_{j}\right)+b .
$$

Here, the LIBSVN toolbox is employed to help implement the prediction based on support vector machine [16].

The working principle of the grid search method is to search all parameters to obtain the optimal parameters. However, this algorithm is simple to implement but it seems to be time consuming. This paper employs an improved grid search method to evaluate the performance of each parameter [17]. In detail, a coarse search is performed in a large range, and a fine search is performed in a small range. Then, all possible parameters are found out and the optimal SVM parameters will be chosen then. The calculation model is shown in Figure 1.

\section{Prediction of Dynamic Wall Pressure for Silos}

The geometric sizes of silos and the physical parameters of storage materials have significant impacts on the dynamic wall pressure of silos. In addition, during silo discharge, the dynamic wall pressure is also different at different locations. Usually, the scale model, numerical simulation, and theoretical analysis are often combined to study the dynamic lateral pressure of silos, and it shows that results of the three methods fit well and can be used as the basis for silo design and dynamic lateral pressure research. Here, to verify the efficiency of the proposed support vector machine-based model on the prediction of the dynamic side pressure prediction model of silos, a database of 505 dynamic side pressures during silo central discharge is collected [18-23].Some relevant parameters are presented in Table 1.

This paper takes the depth of silos, inner diameter, height-diameter ratio, discharge opening width, dip angle of funnel, internal friction coefficient, external friction coefficient, gravity density, and measurement point position as 


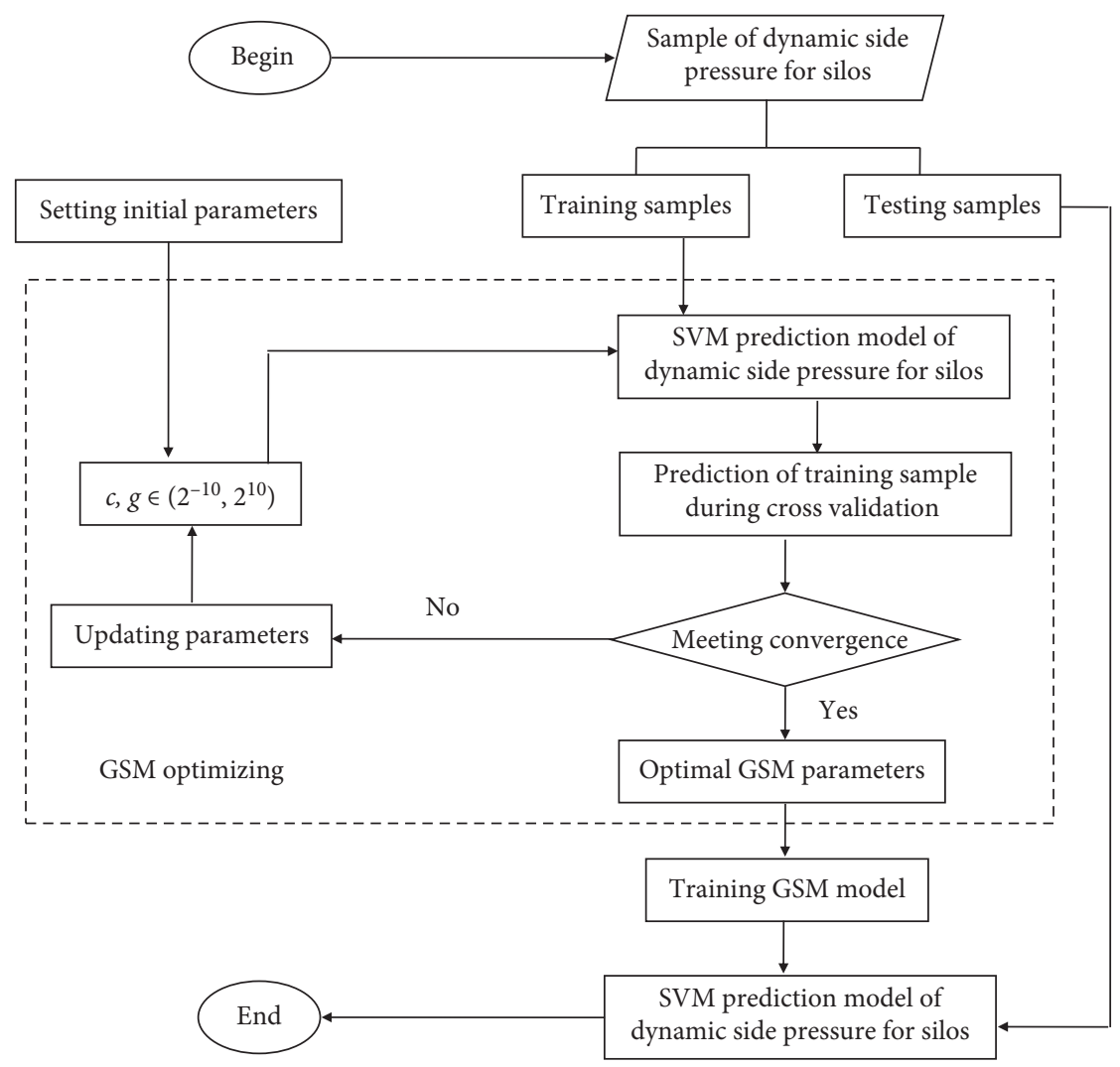

Figure 1: SVM prediction model of dynamic wall pressure for silos.

TABLE 1: Relevant parameters.

\begin{tabular}{|c|c|c|c|c|c|c|c|c|}
\hline Sources & $\begin{array}{l}\text { Depth of } \\
\text { silos (m) }\end{array}$ & $\begin{array}{c}\text { Inner } \\
\text { diameter } \\
(\mathrm{m})\end{array}$ & $\begin{array}{l}\text { Height- } \\
\text { diameter } \\
\text { ratio } \\
\end{array}$ & $\begin{array}{l}\text { Dip angle of } \\
\text { funnel }\left(^{\circ}\right)\end{array}$ & $\begin{array}{c}\text { Width of } \\
\text { discharge } \\
\text { opening }(\mathrm{m})\end{array}$ & $\begin{array}{l}\text { Internal } \\
\text { friction } \\
\text { coefficient }\end{array}$ & $\begin{array}{l}\text { External } \\
\text { friction } \\
\text { coefficient }\end{array}$ & $\begin{array}{l}\text { Gravity } \\
\text { density } \\
\left(\mathrm{kg} / \mathrm{m}^{3}\right) \\
\end{array}$ \\
\hline \multirow{3}{*}{ Zhao [18] } & \multirow{3}{*}{1.2} & \multirow{3}{*}{0.7} & \multirow{3}{*}{1.71} & 50 & 0.1 & 0.3 & 0.3 & \multirow{3}{*}{1000} \\
\hline & & & & 55 & 0.18 & 0.4 & 0.4 & \\
\hline & & & & 60 & 0.26 & 0.5 & 0.5 & \\
\hline \multirow{3}{*}{ Ding [19] } & \multirow{3}{*}{1.2} & \multirow{3}{*}{0.7} & \multirow{3}{*}{1.71} & 55 & 0.12 & 0.25 & 0.35 & \multirow{3}{*}{2000} \\
\hline & & & & 60 & 0.18 & 0.3 & 0.45 & \\
\hline & & & & 60 & 0.24 & 0.35 & 0.55 & \\
\hline \multirow{6}{*}{$\begin{array}{l}\text { Yuan and } \\
\text { Liu [20] }\end{array}$} & \multirow{6}{*}{5} & 1.5 & 2.9 & & 0.3 & \multirow{6}{*}{0.58} & \multirow{6}{*}{0.4} & \multirow{6}{*}{816} \\
\hline & & 2 & 2.2 & & 0.4 & & & \\
\hline & & 2.5 & 1.76 & & 0.5 & & & \\
\hline & & 3 & 1.47 & 50 & 0.6 & & & \\
\hline & & 3.5 & 1.28 & & 0.7 & & & \\
\hline & & 4 & 1.1 & & 0.8 & & & \\
\hline \multirow{3}{*}{ Li [21] } & 5 & 1.5 & 3.3 & & 0.3 & \multirow{3}{*}{0.5} & \multirow{3}{*}{0.4} & \multirow{3}{*}{800} \\
\hline & 3.5 & 1 & 3.5 & 50 & 0.2 & & & \\
\hline & 1.7 & 0.5 & 0.49 & & 0.03 & & & \\
\hline $\begin{array}{l}\text { Wang et al. } \\
{[22]}\end{array}$ & 1 & 0.5 & 2 & 0 & 0.1 & 0.6 & 0 & 970 \\
\hline \multirow{3}{*}{$\begin{array}{l}\text { Chen and } \\
\text { Liang [23] }\end{array}$} & \multirow{3}{*}{3.6} & \multirow{3}{*}{1.5} & \multirow{3}{*}{2.4} & \multirow{3}{*}{60} & \multirow{3}{*}{0.3} & 0.53 & 0.4 & \multirow{3}{*}{800} \\
\hline & & & & & & 0.64 & 0.53 & \\
\hline & & & & & & 0.75 & 0.64 & \\
\hline
\end{tabular}




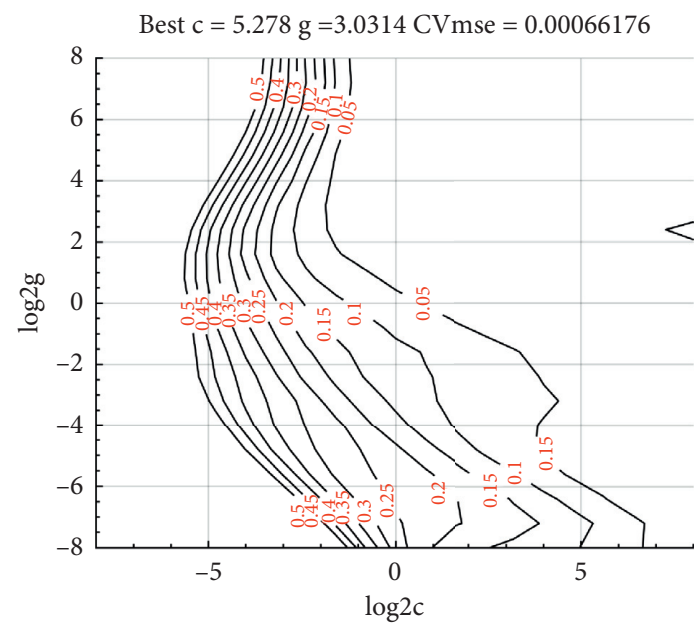

(a)

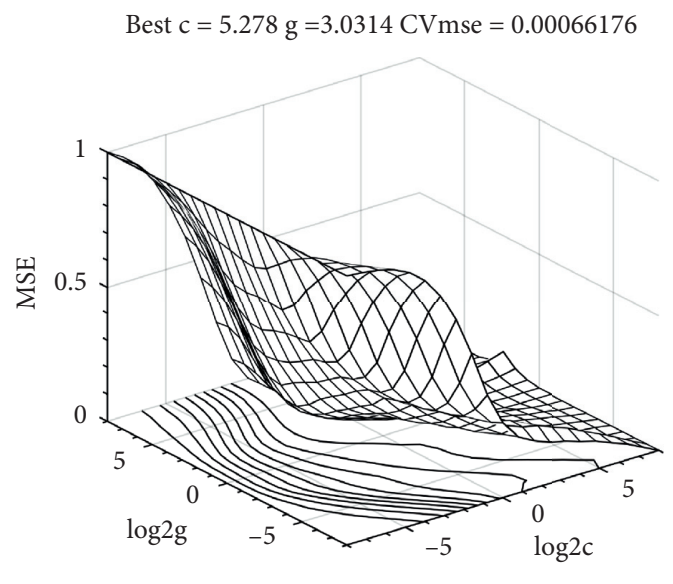

(b)

Figure 2: (a) Fine choosing results of $c$ and $g$ (contour diagram). (b) Fine choosing results of $c$ and $g$ (3D diagram).

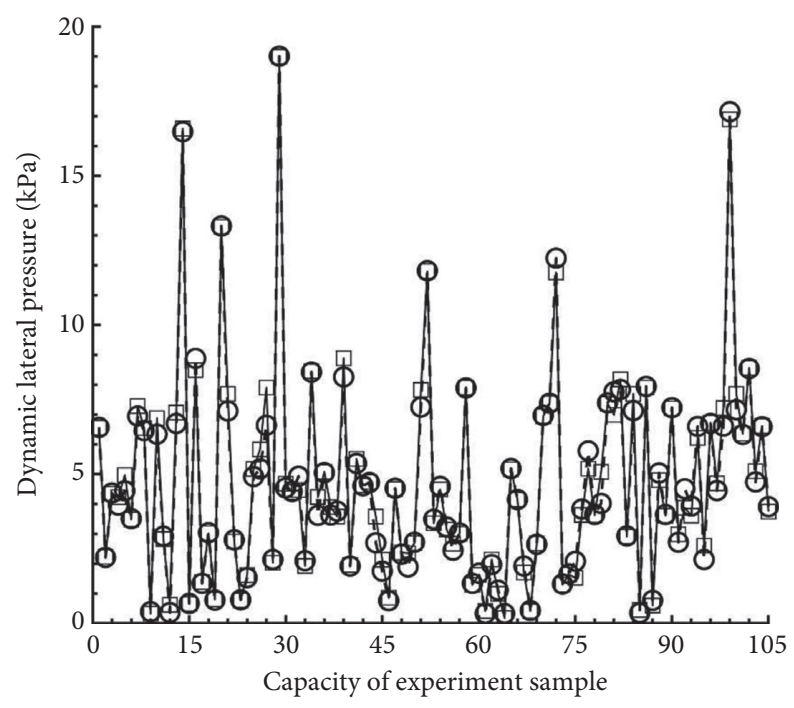

$-\Theta-$ PFC simulation values

$\square$ SVM prediction values

Figure 3: Prediction results of testing samples.

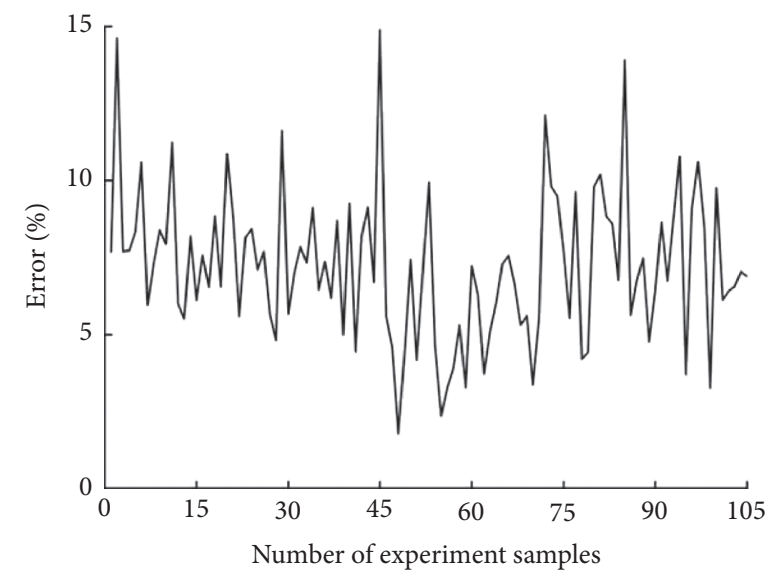

FIgURE 4: Error values. 
TABLE 2: Comparison of the SVM model and BP neural network.

\begin{tabular}{lcc}
\hline & MSE & $R^{2}$ \\
\hline BP neural network & 0.0016884 & 0.95141 \\
SVM method & 0.00041866 & 0.98381 \\
\hline
\end{tabular}

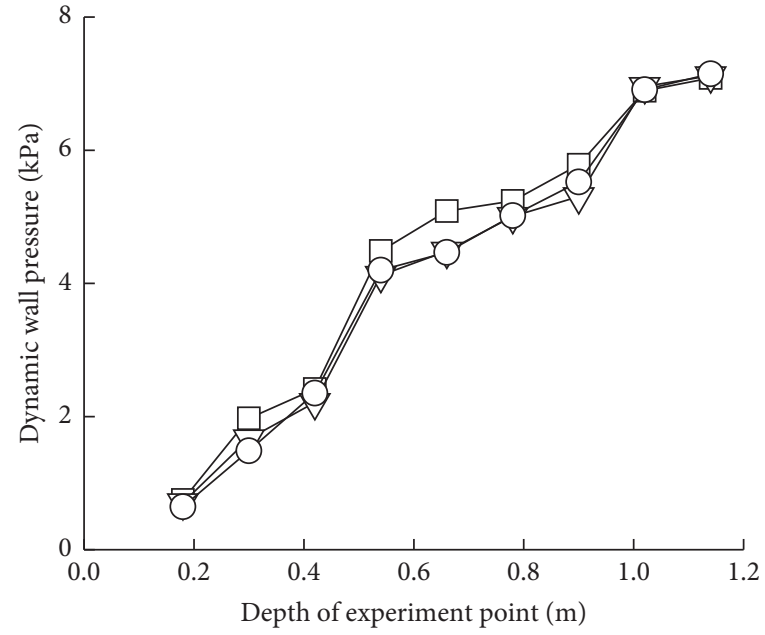

$\neg-$ Experiment values

$-\nabla$ Simulation values

$-\mathrm{O}-$ SVM prediction values

(a)

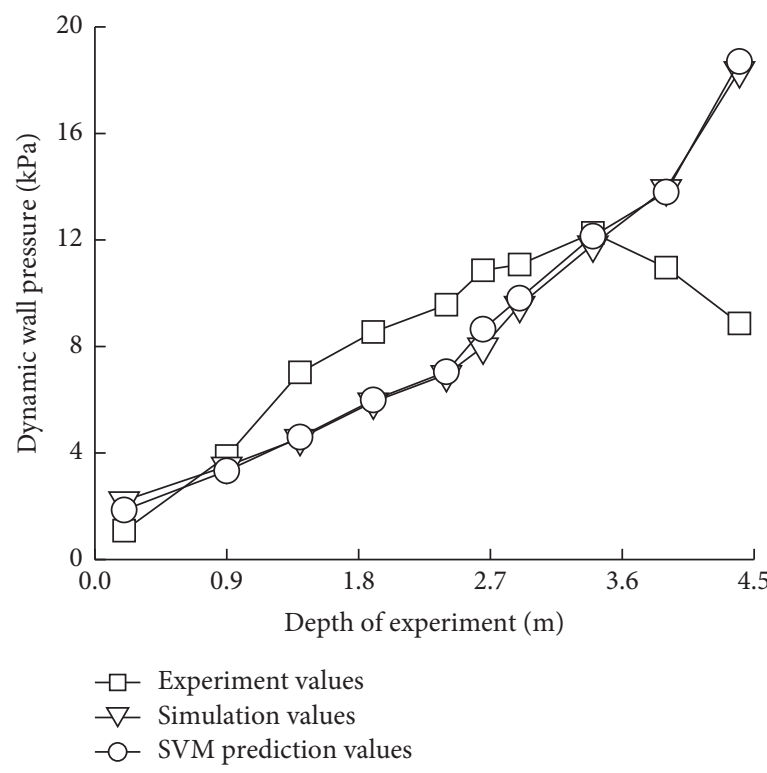

(c)

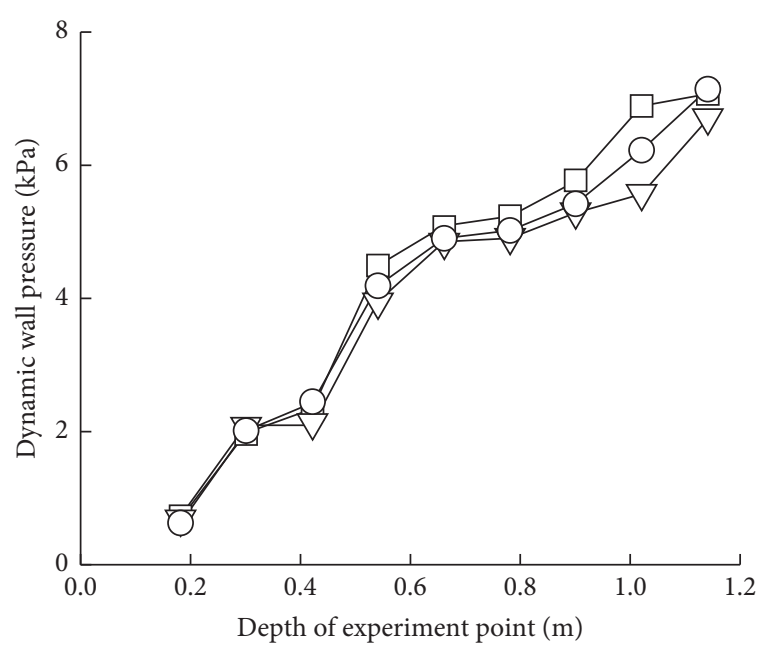

$-\square-$ Experiment values

$-\nabla$ Simulation values

$-O-$ SVM prediction values

(b)

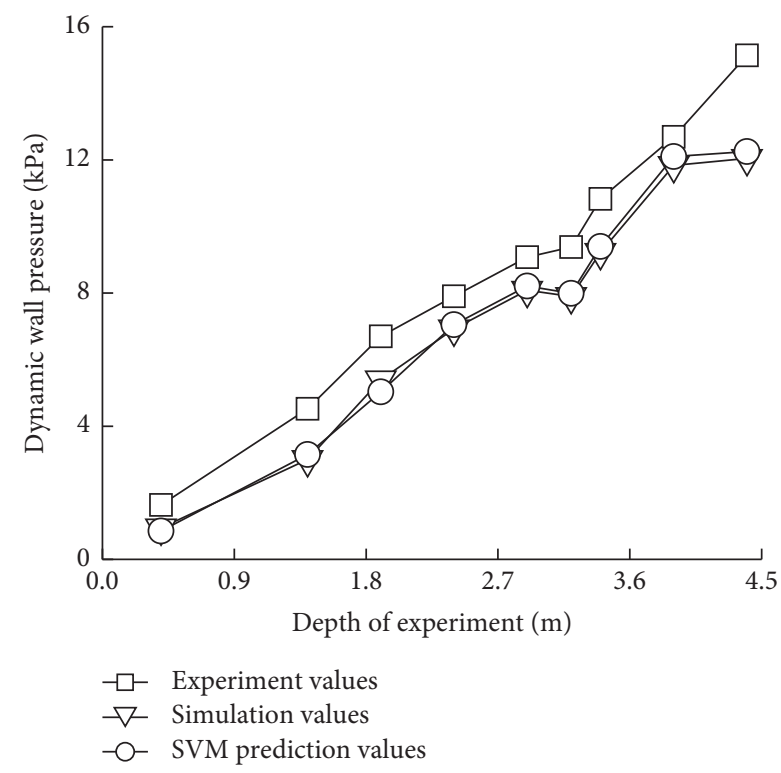

(d)

FIGURE 5: Comparisons with experiment values, simulation values, and prediction values. (a) For literature [18]. (b) For literature [19]. (c) For literature [20]. (d) For literature [21].

input variables and the dynamic wall pressure of silos as the output value. 400 data are randomly selected from the database as training samples, and the remaining 105 data are used as testing samples. The data are described in the form of a matrix, and each row is a set of data, and each column corresponds to a variable. In order to ensure the accuracy and reliability of the prediction model, the original data are normalized as shown in the following equation:

$$
x_{i j}=\frac{x_{i j}-\min _{k}\left(x_{k j}\right)}{\max _{k}\left(x_{k j}\right)-\min _{k}\left(x_{k j}\right)}
$$


where $\max _{k}\left(x_{k j}\right)$ and $\min _{k}\left(x_{k j}\right)$ are the maximum and minimum data, respectively.

The prediction accuracy of the support vector machine (SVM) model is sensitive to the penalty parameter $c$ and kernel parameter $g$. At present, for the support vector machine (SVM) method, the relevant parameters are mainly based on experiences and there is no unified mode. This paper uses the improved grid search method to determine the optimal values of $c$ and $g[24,25]$. The grid optimization program is written by MATLAB program. The parameter optimization process is as follows:

Step 1: the range and step size of $c$ and $g$ are first initialized.

Step 2: $c$ and $g$ are cursorily chosen. The range of $c$ and $g$ is set as $\left[2^{-10}, 2^{10}\right]$. The default values are set as the step sizes of $c$ and $g$, and the default values are also set as the number of cross validations and the step size of the optimal mean square error.

Step 3: $c$ and $g$ are precisely chosen. The range of $c$ and $g$ is set as $\left[2^{-8}, 2^{8}\right] .0 .8$ is set as the step size of $c$ and $g$. 3.0 is set as the number of the cross validations. 0.05 is set as the step size of the optimal mean square error. The fine choosing results are shown in Figure 2.

According to the fine choosing results, 5.278 and 3.0314 are the optimal $c$ and $g$ values, respectively. The optimal $c$ and $g$ values are substituted into the SVM prediction model shown in Figure 1. The mean square error (MSE) and correlation coefficient $\left(R^{2}\right)$ are employed as the evaluating indicators. The prediction results of 105 testing samples are shown in Figure 3. From Figure 3, MSE is 0.00041866 and $R^{2}$ is 0.98381 , which indicate that the testing sample curve basically matches the predicted curve. The errors are shown in Figure 4. The minimum and maximum errors are $1.1 \%$ and $14.88 \%$, and the average relative error is $7.21 \%$.

In order to verify the applicability of the SVM prediction model, the prediction result is compared with BP neural network using the same data, and the results are shown in Table 2. The results show that the support vector machine method can describe well the complex nonlinear relationship between the dynamic side pressure of silos and its influencing factors.

In summary, the proposed SVM prediction model has higher accuracy and can effectively predict the dynamic wall pressure of silos.

\section{Application Analysis}

Relevant parameters in the literature are selected and substituted into the proposed SVM prediction model [18-21]. The results are shown in Figure 5.

It can be seen that the simulated values are slightly larger than the predicted values in the early stage of silo discharge and slightly smaller than the predicted values in the late stage of silo discharge. The prediction accuracy is higher. The testing values are higher than the predicted values. However, with the increment of the depth for measurement points, the dynamic wall pressures are roughly the same. The main reason is that the training samples of the SVM prediction model are from simulated values; therefore, the predicted values are closer to the simulated values. Compared with the PFC program, the proposed method not only improves the calculation efficiency but also can provide a reference for the investigation on dynamic wall pressure when the PFC program cannot calculate the dynamic wall pressure of large silos.

\section{Conclusions}

From this study, some conclusions are presented:

(1) An improved grid search method is used to optimize the punish parameters $(c)$ and kernel function $(g)$ of SVM. The optimal $c$ and $g$ are 5.278 and 3.0314, respectively. The proposed method can avoid the subjective view of relevant parameters based on experiences.

(2) The mean square error (MSE) and the correlation coefficient $\left(R^{2}\right)$ of the SVM prediction model are close to 0 and 1.0, respectively, which indicates that the accuracy of SVM prediction model is satisfied.

(3) The proposed prediction model can effectively solve the nonlinear relationship between the dynamic side pressure of silos and its influencing factors and provide a novel method for the study of the dynamic wall pressure of silos.

\section{Data Availability}

The data used to support the findings of this study are available from the corresponding author upon request.

\section{Conflicts of Interest}

The authors declare that there are no conflicts of interest regarding the publication of this paper.

\section{Acknowledgments}

The authors express their gratitude to the National Natural Science Foundation of China (No. 51578216).

\section{References}

[1] T. Jacek, Confined Granular Flow in Silos, Experimental and Numerical Investigations, Springer, Berlin, China, 2013.

[2] G. Blight, Assessing Loads on Silos and Other Bulk Storage Structures, Taylor \& Francis, New York, NY, USA, 2006.

[3] C. J. Brown and J. Nielsen, Silos: Fundamentals of Theory, Behaviour and Design, E \& FN Spon, London, UK, 1998.

[4] D. L. O. Smith and R. A. Lohnes, Grain Bin Overpres-sures Induced by Dilatancy Upon Unloading, ASAE Paper No.803013, American Society of Agricultural Engineers, St.Joseph, MI, USA, 1980.

[5] L. X. Su, "The elastic deformation and its effect on the wall pressure caused by dischargeing arch in silo," Journal of Zhengzhou Grain College, vol. 18, no. 4, pp. 59-63, 1997. 
[6] H. A. Janssen, "Versucheüber getreidedruck in silozellen," Zeitschriff des Vereines Deutscher Ingenieure, vol. 39, pp. 1045-1049, 1895.

[7] D. H. Liu and J. P. Hao, "Research on lateral pressure upon wall of reinforced concrete silo," Journal of Buliding Structure, vol. 16, no. 5, pp. 57-63, 1995.

[8] F. Yuan, X. Shao, L. M. Wang et al., "A method for calculation lateral pressures on squat silo walls," Engineering Mechanics, vol. 21, no. 3, pp. 96-100, 2004.

[9] S. S. Sun, J. H. Zhao, and C. G. Zhang, "New solution for lateral pressure of silos and bunkers," Journal of Guangxi University (Natural Science Edition), vol. 43, no. 1, pp. 168177, 2018.

[10] J. Zhou, Z. Zhang, M. F. Du et al., "Mesoscopic study on the influence of funnel form changing on silo pressure," Special Sructure, vol. 23, no. 4, pp. 14-16, 2006.

[11] A. Christmann and I. Steinwart, Support Vector Machines, Springer, Berlin, Germany, 2008.

[12] H. Dai, H. Zhang, and W. Wang, "A support vector densitybased importance sampling for reliability assessment," Reliability Engineering \& System Safety, vol. 106, pp. 86-93, 2012.

[13] Y. Wang, X. Zhao, and B. Wang, "LS-SVM and Monte Carlo methods based reliability analysis for settlement of soft clayey foundation," Journal of Rock Mechanics and Geotechnical Engineering, vol. 5, no. 4, pp. 312-317, 2013.

[14] J. W. Wang, Y. S. Xu, and J. X. Li, "Prediction of slope stability coeff icient based on grid search support vector machine," Railway Engineering, vol. 59, no. 5, pp. 312-317, 2019.

[15] S. Weerasinghe, S. Erfani, T. Alpcan et al., "Support vector machines resilient against training data integrity attacks," Pattern Recognition, vol. 96, no. 12, 2019.

[16] C. C. Chang and C. J. Lin, "A library for support vector machines," ACM Transactions on Intelligent Systems and Technology, vol. 2, no. 3, pp. 389-396, 2011.

[17] H. A. Fayed and A. F. Atiya, "Speed up grid-search for parameter selection of support vector machines," Applied Soft Computing, vol. 80, no. 7, pp. 202-210, 2019.

[18] S. Zhao, Analysis and Application of Silo Storage pressure,Doctoral Dissertation, Wuhan University of Technology, Wuhan, China, 2013.

[19] S. W. Ding, "Discrete element analysis of storage side pressure of silo based on PFC3D", M.S. thesis, Wuhan University of Technology, Wuhan, China, 2014.

[20] F. Yuan and H. L. Liu, "Numerical simulation of unloading pressure in deep and shallow warehouses using discrete element method," Journal of Henan University of Technology (Natural Science Edition), vol. 41, no. 1, pp. 68-74, 2020.

[21] J. W. Li, "Study on the numerical simulation of the discharge pressure of the silo considering the influence of the warehouse diameter", M.S. thesis, Henan University of Technology, Zhegnzhou, China, 2018.

[22] S. H. Wang, Z. R. Xiao, and K. J. Liu, "Study on the mesoscopic mechanism of the influence of storage particle size on the unloading flow state and silo wall pressure of silos," Journal of Henan University of Technology(Natural Science Edition), vol. 38, no. 6, pp. 86-90, 2017.

[23] C. B. Chen and X. P. Liang, "Discrete element simulation analysis of silo unloading process," Oils and Food Science and Technology, vol. 16, no. 1, pp. 11-13, 2008.

[24] S. Kostić, N. Vasović, and D. Sunarić, “A new approach to grid search method in slope stability analysis using Box-Behnken statistical design," Applied Mathematics and Computation, vol. 256, no. 4, pp. 425-437, 2015.
[25] C. C. Qi and A. Fourie, "Cemented paste backfill for mineral tailings management: review and future perspectives," Minerals Engineering, vol. 144, no. 12, pp. 1-21, 2019. 